\title{
Rare $B$ decays at LHCb
}

\author{
Albert Puig Navarro*t \\ Universität Zürich (Switzerland) \\ E-mail: albert.puigecern.ch
}

Rare decays are flavour changing neutral current processes that allow sensitive searches for phenomena beyond the Standard Model (SM). In the SM, rare decays are loop-suppressed and new particles in SM extensions can give significant contributions. The very rare decay $B_{s}^{0} \rightarrow \mu^{+} \mu^{-}$is in addition helicity suppressed and constitutes a powerful probe for new (pseudo) scalar particles. Of particular interest are furthermore tests of lepton universality in rare $b \rightarrow s \ell^{+} \ell^{-}$decays. The $\mathrm{LHCb}$ experiment is designed for the study of $b$-hadron decays and ideally suited for the analysis of rare decays due to its high trigger efficiency, as well as excellent tracking and particle identification performance. Recent results from the $\mathrm{LHCb}$ experiment in the area of rare decays are presented, including tests of lepton universality and searches for lepton flavour violation.

EPS-HEP 2017, European Physical Society conference on High Energy Physics

5-12 July 2017

Venice, Italy

*Speaker.

${ }^{\dagger}$ On behalf of the LHCb Collaboration. 


\section{Introduction}

Quarks and leptons in the Standard Model (SM) are organized in flavours. Changes between flavours can only occur through the charged current weak interaction and transitions between same-charge fermions must occur through second order, loop processes [四]. Since the Cabibbo-Kobayashi-Maskawa (CKM) quark mixing matrix [ [], []], which governs transitions between flavours, is found to be approximately diagonal, generation-changing processes are suppressed. As a consequence, processes involving flavour changing neutral currents (FCNC) are predicted to be rare within the SM.

Since many observables related to this type of decays can be predicted in the SM with low theoretical uncertainty, loop-mediated processes allow for a precise indirect access to new degrees of freedom at larger scales and provide excellent complementarity to direct searches of new phenomena. Many models of New Physics (NP) predict significant changes in the measured quantities, making the study of rare decays of flavoured hadrons an ideal laboratory for studying physics beyond the Standard Model.

\section{2. $B \rightarrow \ell^{+} \ell^{-}$decays}

The first type of FCNC to be covered here are the fully leptonic $B \rightarrow \ell^{+} \ell^{-}$decays. These decays are suppressed due to their loop-only diagrams, the involved CKM matrix elements and the particular helicity structure of a pseudoscalar decaying into a pair of leptons, and thus are very rare in the SM. In addition, their branching fractions can be predicted very cleanly thanks to the fact that only one hadronic parameter is needed:

$$
\mathscr{B}\left(B_{q}^{0} \rightarrow \ell^{+} \ell^{-}\right)=\frac{\tau_{B_{q}^{0}} G_{F}^{4} M_{W}^{2} \sin ^{4} \theta_{W}}{8 \pi^{5}}\left|C_{10} V_{t b} V_{t q}^{*}\right| F_{B}^{2} m_{B_{q}^{0}} m_{\ell}^{2} \sqrt{1-\frac{4 m_{\ell}^{2}}{m_{B_{q}^{0}}^{2}}}
$$

which results in the time-integrated predictions of [ [

$$
\begin{aligned}
\mathscr{B}\left(B_{s}^{0} \rightarrow \mu^{+} \mu^{-}\right) & =(3.66 \pm 0.23) \times 10^{-9}, \\
\mathscr{B}\left(B^{0} \rightarrow \mu^{+} \mu^{-}\right) & =(1.06 \pm 0.09) \times 10^{-10} \\
\mathscr{B}\left(B_{s}^{0} \rightarrow \tau^{+} \tau^{-}\right) & =(7.73 \pm 0.49) \times 10^{-7} \\
\mathscr{B}\left(B^{0} \rightarrow \tau^{+} \tau^{-}\right) & =(2.22 \pm 0.19) \times 10^{-8}
\end{aligned}
$$

where the uncertainty mainly comes from the knowledge of the decay constants and the CKM matrix elements.

Culminating a story started more than thirty years ago by the CLEO collaboration [5], the LHCb and CMS collaborations performed a combined analysis of the data collected during Run I, and reported the first observation of $B_{s}^{0} \rightarrow \mu^{+} \mu^{-}$with a significance of $6.2 \sigma$ and an evidence for $B^{0} \rightarrow \mu^{+} \mu^{-}$at $3 \sigma$ [國. The LHCb collaboration has since updated their measurement to add part of the data collected in Run II, up to a total of $4.4 \mathrm{fb}^{-1}$ and to introduce many improvements in the analysis. The obtained results [四]:

$$
\begin{aligned}
& \mathscr{B}\left(B_{s}^{0} \rightarrow \mu^{+} \mu^{-}\right)=\left(3.0 \pm 0.6_{-0.2}^{+0.3}\right) \times 10^{-9}, \\
& \mathscr{B}\left(B^{0} \rightarrow \mu^{+} \mu^{-}\right)<3.4 \times 10^{-10} \text { at } 95 \% \mathrm{CL},
\end{aligned}
$$


constitute the first single experiment observation of the $B_{s}^{0}$ mode and show a $\sim 30 \%$ improvement in the statistical uncertainty (on top of the dataset size increase) in the limit of the $B^{0}$ branching fractions.

Additionally, the $B_{s}^{0} \rightarrow \mu^{+} \mu^{-}$effective lifetime has been measured for the first time, and has been found to be $\tau\left(B_{s}^{0} \rightarrow \mu^{+} \mu^{-}\right)=2.04 \pm 0.44 \pm 0.05 \mathrm{ps}$. This quantity will allow to disentangle scalar and non-scalar NP contributions, which could leave the branching fractions unaffected but modify the relative contributions from the lifetimes of $B_{s, H}$ and $B_{s, L}$ [ [ $]$ ].

While the $B \rightarrow \tau^{+} \tau^{-}$decays are more abundant, they are also more challenging experimentally due to the presence of at least two neutrinos in the final state. The LHCb collaboration has searched for these decays using the $\tau^{-} \rightarrow \pi^{-} \pi^{+} \pi^{-} v$ decay mode [Q].

The analysis exploits the intermediate resonances of the $\tau^{-} \rightarrow a_{1}(1260)^{-} v \rightarrow \rho(770)^{0} \pi^{-} v$ to define several regions in the data, which are used as signal and background, as well as for the training and optimisation of the selection. A two-stage multivariate classifier approach is used: a first neural network (NN) is used to reduce combinatorial background, while a second one is used to obtain the signal yield by means of a fit to its output. No signal is observed, and upper limits for each $B$ meson are obtained [Q],

$$
\begin{aligned}
& \mathscr{B}\left(B_{s}^{0} \rightarrow \tau^{+} \tau^{-}\right)<6.8 \times 10^{-3} \text { at } 95 \% \mathrm{CL}, \\
& \mathscr{B}\left(B^{0} \rightarrow \tau^{+} \tau^{-}\right)<2.1 \times 10^{-3} \text { at } 95 \% \mathrm{CL},
\end{aligned}
$$

by considering that only that particular mode is present, as no separation is possible between $B^{0}$ and $B_{s}^{0}$ in the NN output. These are the best limits obtained to date.

\section{Lepton Flavour Universality}

In the SM, with the exception of the Higgs boson, particles couple equally to different lepton flavours. As a consequence, ratios of decay rates such as

$$
R_{K}=\frac{\mathscr{B}\left(B^{+} \rightarrow K^{+} \mu^{+} \mu^{-}\right)}{\mathscr{B}\left(B^{+} \rightarrow K^{+} e^{+} e^{-}\right)}
$$

are expected to be very close to unity, except from very small Higgs penguin contributions and difference in phase space due to the lepton masses.

In 2014, the LHCb collaboration measured $R_{K}$ in the dilepton mass squared $\left(q^{2}\right)$ range $1<q^{2}<$ $6 \mathrm{GeV}^{2} / c^{4}$ to be $0.745_{-0.074}^{+0.090}$ (stat) \pm 0.036 (syst) [ए0], $2.6 \sigma$ away from the SM prediction of $R_{K}=$ $1.00 \pm \mathscr{O}(0.01)[\square,[2]$. This discrepancy, combined with the $4.0 \sigma$ enhancement of $\tau$ with respect to $\mu$ in tree-level $B^{+} \rightarrow D^{(*)} \ell^{+} v_{\ell}$ decays observed by BaBar, Belle and LHCb [ए3]], prompted great theoretical interest in these types of measurements. Recently, the LHCb collaboration has used $3 \mathrm{fb}^{-1}$ of data collected during 2011 and 2012 to measure

$$
R_{K^{* 0}}=\frac{\mathscr{B}\left(B^{0} \rightarrow K^{* 0} \mu^{+} \mu^{-}\right)}{\mathscr{B}\left(B^{0} \rightarrow K^{* 0} e^{+} e^{-}\right)}
$$

in two bins of $q^{2}$ with different physics contributions: low $\left(0.045<q^{2}<1.1 \mathrm{GeV}^{2} / c^{4}\right)$, dominated by the photon pole, and central $\left(1.1<q^{2}<6 \mathrm{GeV}^{2} / c^{4}\right)$, where the contribution from the $C_{9}^{(\prime)}$ Wilson coefficient becomes more important. 

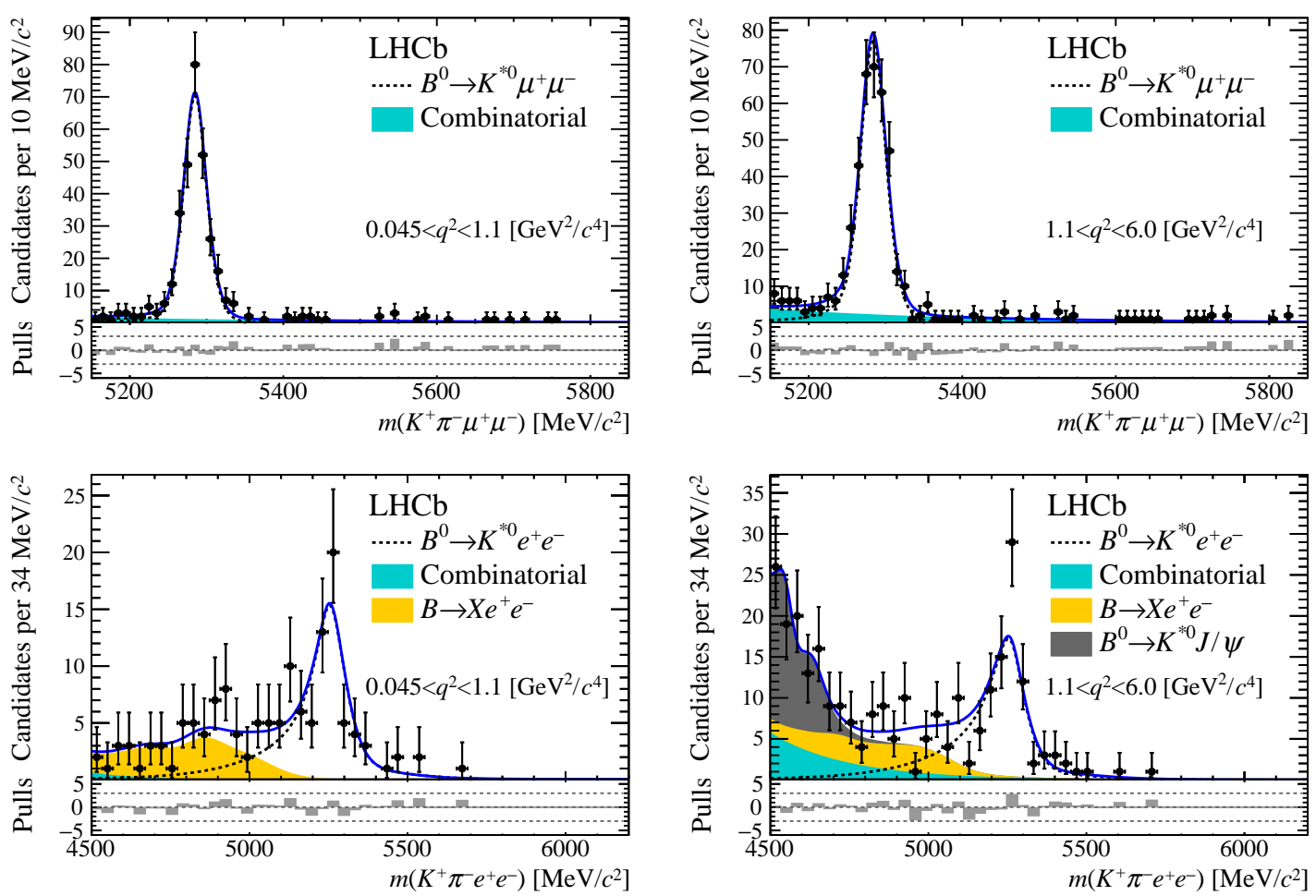

Figure 1: Fit to the $m\left(K^{+} \pi^{-} \mu^{+} \mu^{-}\right)$(top) and $m\left(K^{+} \pi^{-} e^{+} e^{-}\right)$(bottom) invariant mass in the low- (left) and central- $q^{2}$ (right) bins.

In this measurement, it is crucial to control the muon and electron efficiencies with very high precision. To control the systematic uncertainties arising from the differences in reconstruction between decays with muons and electrons, a double ratio strategy is used, in which the (resonant) $B^{0} \rightarrow K^{* 0} J / \psi\left(\rightarrow \ell^{+} \ell^{-}\right)$modes are used in conjunction with the rare (non-resonant) modes.

A fit to the $B^{0}$ invariant mass, shown in Fig. 四, is performed to determine the yields of the four studied modes, with the shape of the distribution for the electron modes being obtained separately according to the number of bremsstrahlung photons recovered. The fit is performed simultaneously in categories defined by how the rare electron mode was triggered-either using the electrons, the hadrons or other particles in the event—and the likelihoods for each category are combined to obtain [14]]

$$
\begin{aligned}
R_{K^{* 0}}\left(\text { low }-q^{2}\right) & =0.66_{-0.07}^{+0.11} \pm 0.03, \\
R_{K^{* 0}}\left(\text { central }-q^{2}\right) & =0.69_{-0.07}^{+0.11} \pm 0.05,
\end{aligned}
$$

compatible with the SM expectations at $2.1-2.3$ and $2.4-2.5$ standard deviations for the lowand high $-q^{2}$ regions, respectively.

\section{Conclusions}

Searches for NP with rare $B$ decays performed at LHCb have pushed the SM to the limit, and interesting tensions have appeared as a consequence. 
On one hand, the study of $B \rightarrow \ell^{+} \ell^{-}$decays at LHCb has pushed the boundaries of experimental knowledge, with the first single-experiment observation of $B_{s}^{0} \rightarrow \mu^{+} \mu^{-}$and the world's best limit on the extremely challenging $B \rightarrow \tau^{+} \tau^{-}$; these results are so far compatible with the SM, but more precision is still needed. Additionally, the first measurement of the $B_{s}^{0} \rightarrow \mu^{+} \mu^{-}$lifetime opens a door to a new type of measurements that will lead to more stringent constraints on NP.

On the other hand, the measurements of $B^{+} \rightarrow K^{+} \ell^{+} \ell^{-}$and $B^{0} \rightarrow K^{* 0} \ell^{+} \ell^{-}$decays performed at $\mathrm{LHCb}$ are showing hints of lepton flavour universality violation. These, combined with other tensions observed in $b \rightarrow s \mu^{+} \mu^{-}$decays and in tree-level $B^{+} \rightarrow D^{(*)} \ell^{+} v_{\ell}$ decays, paint a very interesting picture in the search for NP in the near future. Given the latest results presented here, which strengthen the tensions with the SM, LHCb is pursuing a wide program of measurements of lepton flavour universality in $b \rightarrow s \ell^{+} \ell^{-}$decays, with further $R_{X}$ results, as well as the measurement of angular distributions, with the aim of confirming or disproving these hints in the following years.

\section{References}

[1] S. L. Glashow, J. Iliopoulos and L. Maiani, Weak Interactions with Lepton-Hadron Symmetry, Phys. Rev. D2 (Oct, 1970) 1285-1292.

[2] N. Cabibbo, Unitary Symmetry and Leptonic Decays, Phys. Rev. Lett. 10 (Jun, 1963) 531-533.

[3] M. Kobayashi and T. Maskawa, CP-Violation in the Renormalizable Theory of Weak Interaction, Prog. Theor. Phys. 49 (1973) 652-657.

[4] C. Bobeth, M. Gorbahn, T. Hermann, M. Misiak, E. Stamou and M. Steinhauser, $B_{s, d} \rightarrow l^{+} l^{-}$in the Standard Model with Reduced Theoretical Uncertainty, Phys. Rev. Lett. 112 (2014) 101801, [1311.0903].

[5] CLEO collaboration, P. Avery et al., Upper Limit on Flavor Changing Neutral Current Decays of the $b$ Quark, Phys. Rev. Lett. 53 (1984) 1309-1313.

[6] CMS AND LHCb COLLABORATIONS collaboration, V. Khachatryan et al., Observation of the rare $B_{s}^{0} \rightarrow \mu^{+} \mu^{-}$decay from the combined analysis of CMS and LHCb data, Nature $\mathbf{5 2 2}(2015) 68$ CERN-PH-EP-2014-220, CMS-BPH-13-007, LHCb-PAPER-2014-049, [1411.4413].

[7] LHCB collaboration, R. Aaij et al., Measurement of the $B_{s}^{0} \rightarrow \mu^{+} \mu^{-}$branching fraction and effective lifetime and search for $B^{0} \rightarrow \mu^{+} \mu^{-}$decays, Phys. Rev. Lett. $118(2017) 191801$ LHCb-PAPER-2017-001, CERN-EP-2017-041, [ए]703.05747].

[8] K. De Bruyn, R. Fleischer, R. Knegjens, P. Koppenburg, M. Merk, A. Pellegrino et al., Probing New Physics via the $B_{s}^{0} \rightarrow \mu^{+} \mu^{-}$Effective Lifetime, Phys. Rev. Lett. 109(2012) 041801, [1204.1737]].

[9] LHCB collaboration, R. Aaij et al., Search for the decays $B_{s}^{0} \rightarrow \tau^{+} \tau^{-}$and $B^{0} \rightarrow \tau^{+} \tau^{-}$, Phys. Rev. Lett. 118 (2017) 251802 LHCb-PAPER-2017-003, CERN-EP-2017-034, [ए103.02508].

[10] LHCB collaboration, R. Aaij et al., Test of lepton universality using $B^{+} \rightarrow K^{+} \ell^{+} \ell^{-}$decays, Phys. Rev. Lett. 113 (2014) 151601 LHCb-PAPER-2014-024, CERN-PH-EP-2014-140, [1406.6482].

[11] C. Bobeth, G. Hiller and G. Piranishvili, Angular distributions of $\bar{B} \rightarrow \bar{K} \ell^{+} \ell^{-}$decays, पHEP12] [2007) 040, [0709.4174].

[12] M. Bordone, G. Isidori and A. Pattori, On the Standard Model predictions for $R_{K}$ and $R_{K^{*}}$, Eur. Phys. [.C76(2016) 440, [11605.07633]. 
[13] Heavy Flavor Averaging Group collaboration, Y. Amhis et al., Averages of b-hadron, c-hadron, and $\tau$-lepton properties as of summer 2014, 1412.7515 .

[14] LHCB collaboration, R. Aaij et al., Test of lepton universality with $B^{0} \rightarrow K^{* 0} \ell^{+} \ell^{-}$decays, एसEP08 [2017)055 LHCb-PAPER-2017-013, CERN-EP-2017-100, [1705.05802]. 